EPJ Web of Conferences 45, 01008 (2013)

DOI: $10.1051 /$ epjconf/20134501008

C Owned by the authors, published by EDP Sciences, 2013

\title{
Active Control of Flow around NACA 0015 Airfoil by Using DBD Plasma Actuator
}

\author{
Y.E. Akansu ${ }^{1}$, F. Karakaya ${ }^{2}$, and A. Şanlısoy ${ }^{1}$ \\ ${ }^{1}$ Department of Mechanical Engineering, Faculty of Engineering, Nigde University, Nigde, Turkey \\ ${ }^{2}$ Department of Electrical and Electronics Engineering, Faculty of Engineering, Nigde University, Nigde, Turkey
}

\begin{abstract}
In this study, effect of plasma actuator on a flat plate and manipulation of flow separation on NACA0015 airfoil with plasma actuator at low Reynolds numbers were experimentally investigated. In the first section of the study, plasma actuator which consists of positive and grounded electrode couple and dielectric layer, located on a flat plate was actuated at different frequencies and peak to peak voltages in range of 3-5 kHz and 6-12 kV respectively. The induced air flow velocity on the surface of flat plate was measured by pitot tube at different locations behind the actuator. The influence of dielectric thickness and unsteady actuation with duty cycle was also examined. In the second section, the effect of plasma actuator on NACA0015 airfoil was studied at Reynolds number 15000 and 30000. Four plasma actuators were placed at $\mathrm{x} / \mathrm{C}=0.1,0.3,0.5$ and 0.9 , and different electrode combinations were activated by sinusoidal signal. Flow visualizations were done when the attack angles were $0^{\circ}, 5^{\circ}, 10^{\circ}, 15^{\circ}$ and $20^{\circ}$. The results indicate that up to the $15^{\circ}$ attack angle, the separated flow was reattached by plasma actuator at $12 \mathrm{kV}$ peak to peak voltage and 4 $\mathrm{kHz}$ frequency. However, $12 \mathrm{kV}_{\mathrm{pp}}$ voltage was insufficient to reattach the flow at $20^{\circ}$ angle of attack. The separated flow could be reattached by increasing the voltage up to $13 \mathrm{kV}$. Lift coefficient was also increased by the manipulated flow over the airfoil. Results showed that even high attack angles, the actuators can control the flow separation and prevent the airfoil from stall at low Reynolds numbers.
\end{abstract}

\section{Introduction}

Elimination or reduction of the effects of flow induced problem can be achieved by the application of the various active and passive flow control methods. Passive methods mean adding various devices to the system without using any additional auxiliary power. In active methods, it is necessary to have an external energy input such as blowing and suction from a surface, acoustic excitation, periodic rotation or oscillation, wall motion, and electromagnetic forces could be given as examples. In electrical control of the flow, local ionized air, produced as a result of electrical discharge, drives the bulk motion of neutral molecules which is called Electrohydrodynamic (EHD). EHD is useful as a flow control mechanism, because electrical energy is converted to momentum without any need of moving mechanical parts.

It is a promising method for aero industrial applications because of adequate response time, sample structure, and many features such as controlling the separation, increasing the lift forces, decreasing the drag forces, delaying the stall angle. As a result of these beneficial features, many researchers have been in a great interest on EHD plasma actuators. On the other hand, more enhancements are still necessary to achieve effective flow control at high Reynolds number for the industrial applications.

Roth et al.[1-3] has shown the ability of plasma actuator by accelerating the neutral flow up to approximately $3 \mathrm{~m} / \mathrm{s}$, flow reattachment on NACA0015 airfoil at $2.85 \mathrm{~m} / \mathrm{s}$ free stream velocity. They divided the actuation physics to paraelecetic which actuates all the actuators at same time and peristaltic flow acceleration which actuates the actuators with traveling electrostatic wave at different phase angle. Also they tried to combine paraelectric and peristaltic effects on the same actuator panel.

Thomas et al.[4] investigated the effects of dielectric material and thickness, applied voltage amplitude and frequency, voltage waveform, exposed electrode geometry, covered electrode width, and multiple actuator arrays. They examined quartz, Teflon, Delrin, and Macor ceramic dielectric material with different thicknesses. High dielectric strength, low dielectric constant and increased thickness of dielectric material, like quartz, caused an improvement in the body force compared with Kapton dielectric material. They showed that body force increases linearly with increasing applied voltage as 
frequency is reduced. They tried serrated edge to demonstrate the effect of electrode geometry which provides a considerable increase in body force over that produced by the same actuator with a conventional electrode at typical operation voltages. However, near saturation voltage the effect of serrate edge was little. They also showed that for optimum body force the covered electrode width should be sufficient that plasma forming is not constrained and they also showed that for multi-actuator arrays, the total body force increases with the number of actuators but does not sum linearly.

Sosa and Artana [5] investigated the effect of plasma actuator on the flow around NACA 0015 airfoil at different flow conditions for variety of velocity and attack angle. They found that for low Re number, the effects of the actuation depend on the power added to the flow and on the relative distance between the actuator and the separation line. They also defined a power coefficient to evaluate the intensity of actuation.

Little et al. [6] investigated the effect of plasma actuator which driven with modulated signals on flap of airfoil at Reynolds number 250000-750000. They showed that amplifying the natural instability associated with vortex shedding from the flap shoulder by modulated waveform was most effective for increasing lift and reducing the time-averaged recirculation region on the flap. They compared the effects of burst modulated and amplitude modulated signals. In the same study it is shown that both the effectiveness and efficiency of the actuator for exciting at low frequencies can be enhanced using a square-wave modulation signal (BM) instead of a sinusoidal signal (AM) and each flap angle is found to have a preferred duty-cycle range for BM that increases with increasing flap angle.

Bernard and Moreau [7] investigated the electrical characteristics of plasma actuator when the actuator has excited by modulated signals. They tried burst modulation, superposition modulation and ring modulation. The induced velocity fluctuation frequencies could be changed by modulation of signals. Bernard et al. [8] examined the effect of plasma actuator to NACA0015 airfoil aerodynamic performance with steady and unsteady actuations. They demonstrated that the plasma actuator improves the lift coefficient, reduces the drag coefficient and delays the stall angle.

Jolibois et al.[9] optimizes the actuator location along the chord. They showed reduction in power consumption by duty cycles. They located seven electrodes along the chord and examined the effect of each actuator to reattach the airflow naturally separated for angles of incidence up to $17^{\circ}$.

Goksel et al.[10] examined the control of air flow separation and aerodynamic performances using plasma actuator at Reynolds numbers $20,500 \leq R e \leq 50,000$ on Eppler E338 airfoil by steady and unsteady actuations. They demonstrated that lift coefficient is increased by decreasing duty cycle, and hence the input power, for duty cycles $3 \%$. Even lower duty cycles, around $0.66 \%$, it was sufficient for effective separation control, corresponding to power inputs on the order of $1.2 \mathrm{~mW}$ per centimeter. In first part of the study, the effect of plasma actuator on the induced airflow was examined on a flat plate by using single DBD actuator. Effect of the dielectric material thickness, voltage, frequency and signal modulations were investigated to find the appropriate actuation to improve the induced airflow velocity on the surface. In the second part of the study, aforementioned actuation parameters were applied for flow control around NACA0015 airfoil. The number of the actuators was also configured as activating the first one and all of the four actuators.

\section{Experimental Setup}

This study has two parts of experimental stages. First part was performed to define properties of plasma induced velocity by the actuator on the flat plate without the free stream velocity. The second part was performed to exert the effect of actuator on the active control of flow around an airfoil. In the experiments, two different high voltage power amplifiers were used to produce atmospheric plasma. First high voltage power amplifier is custommade and has four channels which each one has an audio power amplifier and a step-up transformer. It has a good response for sinusoidal output signal at the broad range of voltages and frequencies in this study. The other power supply utilized is TREK 20/20C-HS model high-voltage power amplifier which uses all solid state technology for high slew rate of $800 \mathrm{~V} / \mu \mathrm{s}$, wide bandwidth, and lownoise operation. The applied voltage and current to the actuator were measured by Tektronix P6015A high voltage probe and Fluke 80i-110s AC/DC current probe and Tektronix TDS2012B oscilloscope was used for signal visualization.

\subsection{Flat plate measurements}

Experiments for flat plate were carried out in a wind tunnel test section of $570 \mathrm{~mm} \times 570 \mathrm{~mm}$ without free stream velocity. The tunnel airflow exit channel was closed by a damper to provide no extraneous airflow in the test section. The actuators located on a $200 \mathrm{~mm}$ wide $250 \mathrm{~mm}$ long Plexiglas flat plate as shown in figure 1.

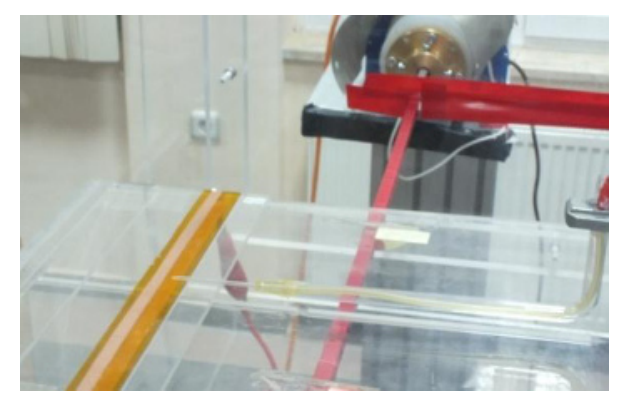

Fig. 1. Illustration of plasma actuator on the flat plate and pitot tube location.

The actuator consists of two, $7 \mathrm{~mm}$ wide and $180 \mathrm{~mm}$ long copper electrodes, and a Kapton tape dielectric material between them. The actuator was excited by the custom-made power amplifier in the ranges of 7-12 kV peak-peak voltages and $3-5 \mathrm{kHz}$ frequencies. To measure the plasma induced velocity, a pitot tube was used to 
obtain dynamic pressures over the plate surface. It was mounted on a computer controlled traverse mechanism and placed behind the actuator at different distances on $\mathrm{x}$ and y axes. The mean pressure was measured by Omega PX278-01D5V pressure transducer and converted to velocity.

\subsection{NACA0015 airfoil measurements}

NACA0015 airfoil geometry was tested in an open low speed wind tunnel with a square test section of $570 \mathrm{~mm} \times$ $570 \mathrm{~mm}$ and free stream velocity range of $0-15 \mathrm{~m} / \mathrm{s}$. Reynolds numbers based on chord length (C) were 15000 and 30000, corresponding to free-stream velocities of 1.9 $\mathrm{m} / \mathrm{s}$ and $3.8 \mathrm{~m} / \mathrm{s}$, respectively. Airfoil geometry has 150 $\mathrm{mm}$ chord length and $570 \mathrm{~mm}$ span length. Two end plates of $280 \mathrm{~mm}$ in diameter were used and spanwise length between them was $500 \mathrm{~mm}$. The actuators were located on NACA0015 airfoil at $\mathrm{x} / \mathrm{C}=0.1,0.3,0.5$ and 0.9 as shown in figure 2. Each actuator consists of 495 $\mathrm{mm}$ grounded and exposed electrodes and Kapton dielectric tape in $130 \mu \mathrm{m}$ thickness between them. Plasma was generated by using custom-made power amplifier at sinusoidal output signal in the ranges of 6 to $13 \mathrm{kV}$ peak to peak voltage at $4 \mathrm{kHz}$ frequency. TREK 20/20C-HS model amplifier were also used at the duty cycle experiments in the case of one actuator located at $\mathrm{x} / \mathrm{C}=$ 0.1 .

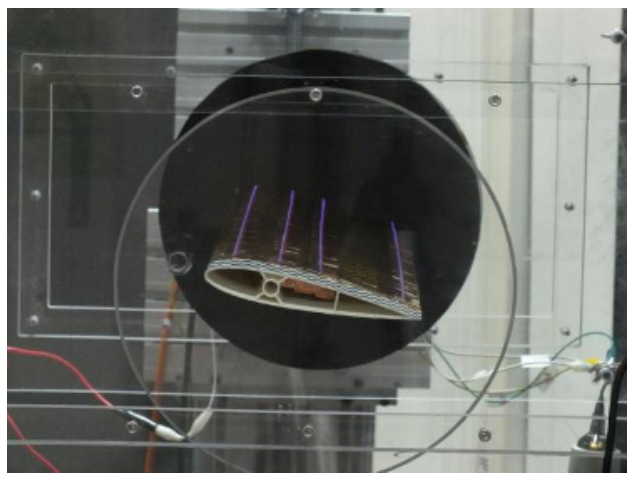

Fig. 2. Schema of plasma actuator on NACA 0015 airfoil.

The airfoil was mounted on a custom-made sixcomponent force-balance system. It is composed of ATI Gamma DAQ F/T load cell transducer and ISEL ZD30 rotary unit which is controlled by computer to adjust attack angle of the airfoil. Smoke-wire system was used to generate smoke in the wind tunnel section. Top plane of test section was lightened by KRUSS OPTRANIC cold light generator to visualize the flow around the airfoil and Fujifilm HS20EXR camera was used to capture the flow visualizations.

\section{Results}

\subsection{Plasma Induced Velocity on the Flat Plate}

Firstly, the effects of voltage and frequencies on the plasma induced flow were investigated on the flat plate. The actuator was excited around optimum frequency and voltage values to examine the effect of each parameter. As seen in figure 3 , the plasma actuator was excited at 4 $\mathrm{kHz}$ frequency with different voltages and as a result a very thin wall jet produced on the surface. It is clearly seen that plasma induced velocity increased by increasing the voltage. At $8 \mathrm{kV}_{\mathrm{pp}}$ applied voltage, induced velocity of $2 \mathrm{~m} / \mathrm{s}$ was observed.

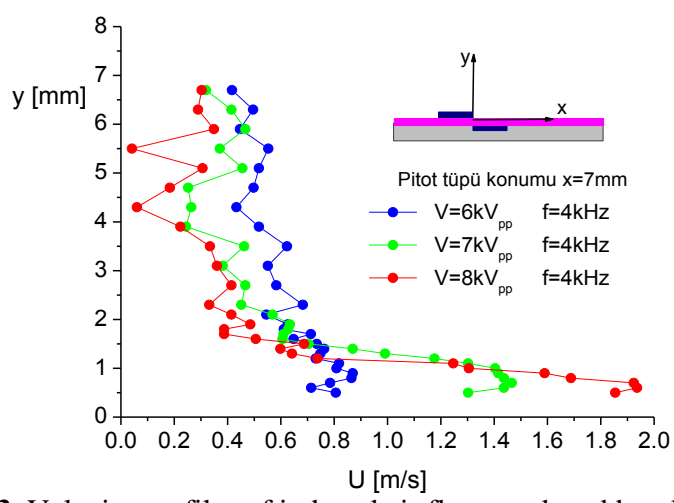

Fig. 3. Velocity profiles of induced air flow produced by plasma actuator versus y axis at $6,7,8 \mathrm{kV}$ voltages and $4 \mathrm{kHz}$ frequency.

By increasing the voltage, plasma regime changed from glow discharge to arc. To avoid the arc generation, double layer dielectric material was used in the other case as seen in figure 4 . In this case it was possible to work at high voltages, but threshold voltage was higher to generate the plasma on the surface. Consequently, the velocity was approximately $1.3 \mathrm{~m} / \mathrm{s}$ at the excitation of 8 $\mathrm{kV}_{\mathrm{pp}}$ and $4 \mathrm{kHz}$. Figure 4 illustrates the result obtained when the actuator was excited at $4 \mathrm{kHz}$ frequency with different voltages from $8 \mathrm{kV}_{\mathrm{pp}}$ to $12 \mathrm{kV}_{\mathrm{pp}}$. We were able to go to higher voltages as a result of double layer dielectric and it let us to reach a higher flow velocity. When the voltage was $12 \mathrm{kV}_{\mathrm{pp}}$, the plasma induced velocity was approximately $3.3 \mathrm{~m} / \mathrm{s}$.

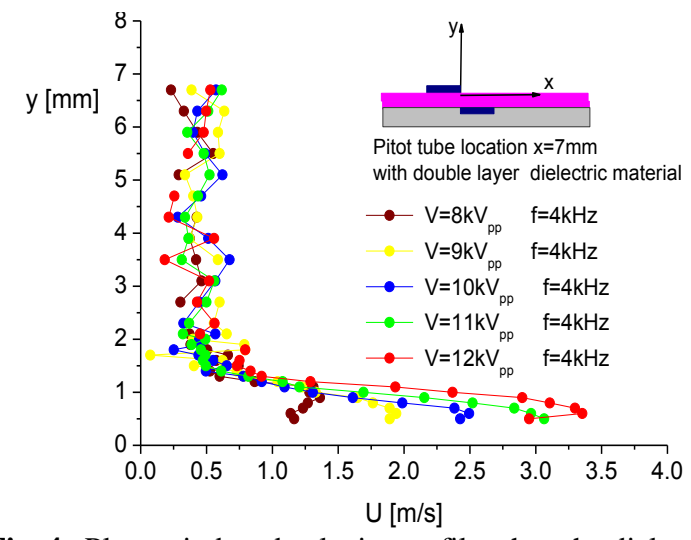

Fig. 4. Plasma induced velocity profile when the dielectric layer was double.

Figure 5 shows the effect of the frequency at a constant voltage of $8 \mathrm{kV}_{\mathrm{pp}}$. There was a considerable change in the flow velocity between the frequencies of 3.5 and $4 \mathrm{kHz}$. When the frequency was $4 \mathrm{kHz}$, the plasma induced velocity was $2 \mathrm{~m} / \mathrm{s}$. However, increasing frequency beyond the $4 \mathrm{kHz}$ was not effective to increase the plasma induced velocity. 
The effects of duty cycles on plasma induced velocity were obtained in the figure 6 . It is clearly seen that the maximum induced velocity obtained when the duty cycle was $100 \%$. When the duty cycle was $30 \%$ the induced velocity was approximately $0.8 \mathrm{~m} / \mathrm{s}$. It must be kept in mind that the power consumption of the plasma actuator decreases as the duty cycles of the signal is reduced.

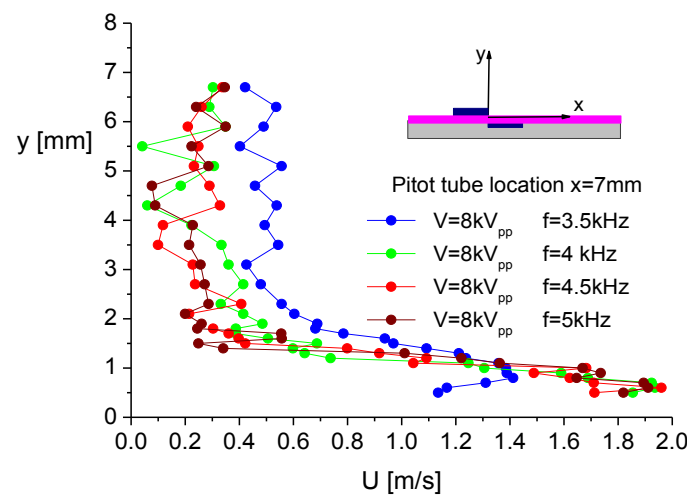

Fig. 5. Velocity profiles of induced air flow produced by plasma actuator versus y axis at $3.5,4,4.5,5 \mathrm{kHz}$ frequencies and $8 \mathrm{kV}_{\mathrm{PP}}$ voltage.

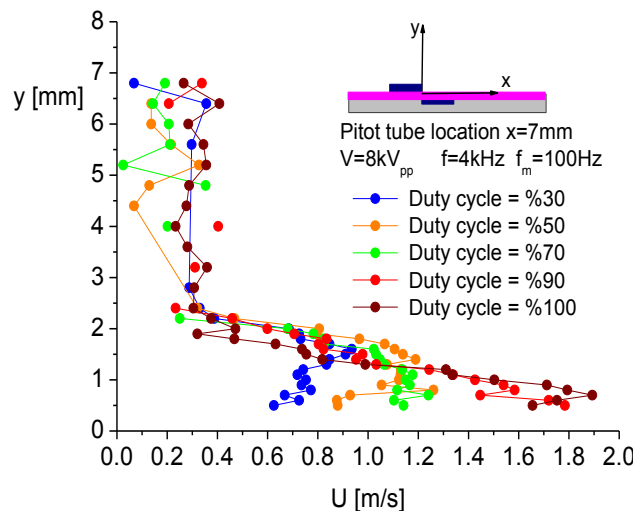

Fig. 6. Effect of duty cycles to plasma induced velocity at $\mathrm{V}=$ $8 \mathrm{kV}_{\mathrm{pp}}, \mathrm{f}=4 \mathrm{kHz}$ and $\mathrm{f}_{\mathrm{m}}=100 \mathrm{~Hz}$.

Pitot tube measurement location is an important parameter for the magnitude of velocity. When the pitot tube is close to plasma actuator the velocity value is higher. The velocity was decreasing with moving the pitot tube away from the plasma actuator as seen in figure 7. For the location of pitot tube at $4 \mathrm{~mm}, 7 \mathrm{~mm}$ and $10 \mathrm{~mm}$, the measured velocities were $1.3,1.6$ and $2.75 \mathrm{~m} / \mathrm{s}$, respectively.

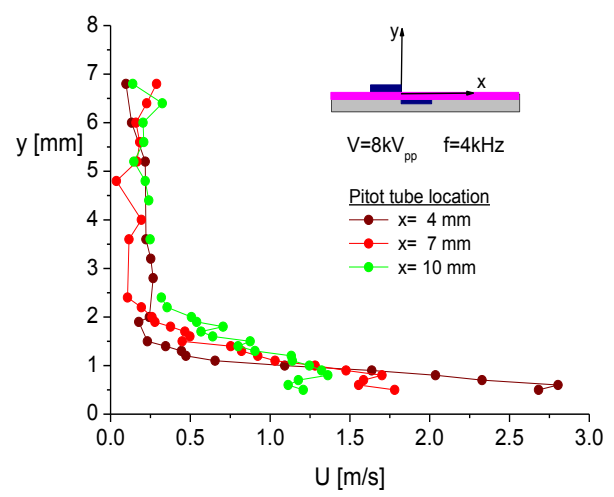

Fig. 7. Effect of pitot tube location on plasma induced velocity.

\subsection{NACA0015 Airfoil Results}

The flow control by plasma actuator was done around NACA0015 airfoil geometry. The aerodynamic force measurements were performed to reveal the effect of plasma actuator on the airfoil. Four actuators, placed on the airfoil, were actuated at $3.5 \mathrm{kHz}$ frequency and $6 \mathrm{kV}$ peak to peak voltage. By using the plasma actuators, the stall angle was delayed from $6^{\circ}$ to $10^{\circ}$ compared with base airfoil (no plasma) at $\operatorname{Re} 30000$ as shown in figure 8 . At the attack angle of $10^{\circ}$, the lift coefficient was increased approximately $100 \%$ where its value increased from 0.5 to 1.0 .

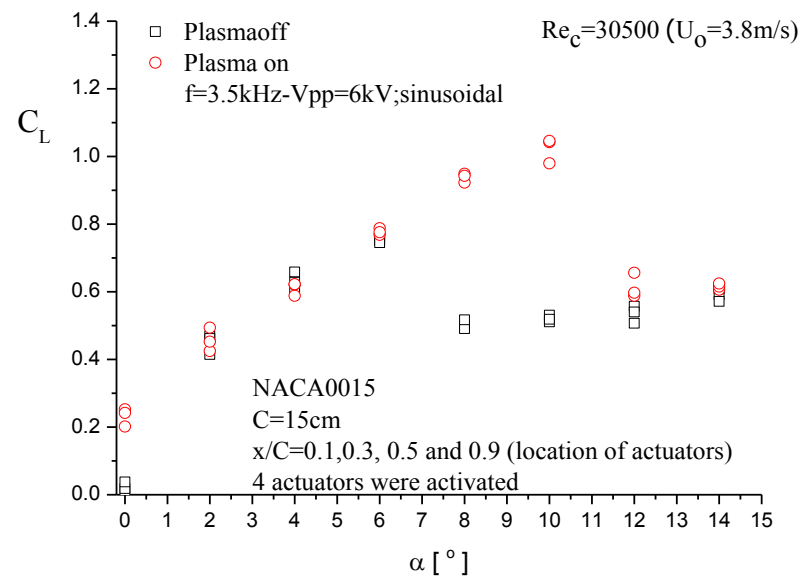

Fig. 8. Lift coefficient versus attack angle when the 4 actuators were active at $\operatorname{Re}=30500$.

The effect of frequency on the lift coefficient at $\alpha=10^{\circ}$ was examined by changing the frequency from $2.5 \mathrm{kHz}$ to $5 \mathrm{kHz}$ as seen in figure 9. Results were shown that the lift coefficient was increased by increasing the frequency.

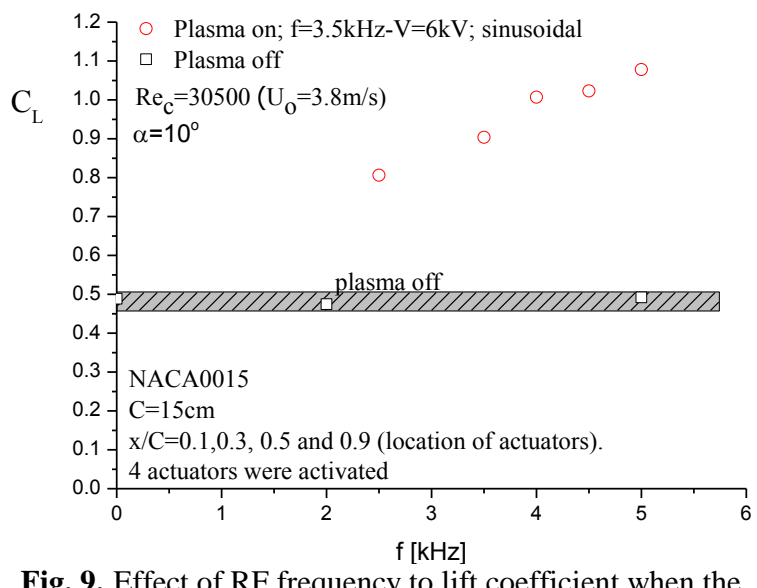

Fig. 9. Effect of RF frequency to lift coefficient when the voltage was $\mathrm{V}=6 \mathrm{kVpp}$.

Influence of the plasma under increased voltage on the lift coefficient was also studied. Figure 10 shows the effect of the voltage for the cases of differently activated actuator configurations. At the lowest voltage value of $5 \mathrm{kV}$, plasma was weak and all activated actuator configurations had same effects on the lift coefficient. In the case of 6 and $7 \mathrm{kV}$, four activated actuator configuration was more effective to increase the lift 
coefficient than the other configurations. When the voltage was over $8 \mathrm{kV}$, the lift coefficient was reached to 1.1 , which is approximately same value with actuation of one or multiple actuators. It is obvious that the number of actuators activated has great effect on the power consumption. For the highest voltage, first actuator near the separation point is enough to provide the same effect by using the lowest power.

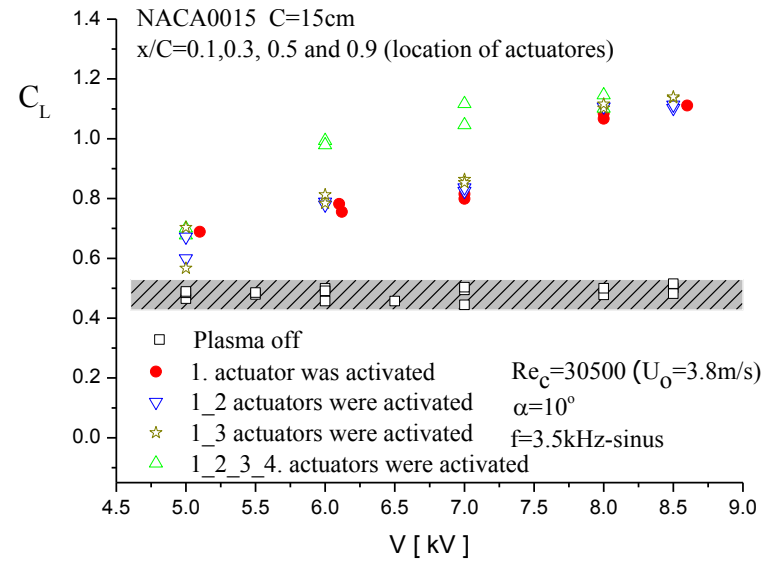

Fig. 10. Effect of voltage and active actuators to lift coefficient when the $\mathrm{RF}$ frequency was $\mathrm{f}=3.5 \mathrm{kHz}$

In figure 11, the plasma actuator was actuated with different percentage of duty cycles at excitation frequencies of $100 \mathrm{~Hz}$ and $17 \mathrm{~Hz}$. The results showed that it was possible to obtain the same lift coefficient by consuming less amount of energy. The excitation frequency also effects the lift coefficient. At the frequency of $100 \mathrm{~Hz}$, the lift coefficient was increased by $25 \%$ for the duty cycle rates between $10-90 \%$ comparing with continuous sinusoidal signal (100\% duty cycle). Even the duty cycle of $10 \%$ increased the lift coefficient more than the continuous signal.

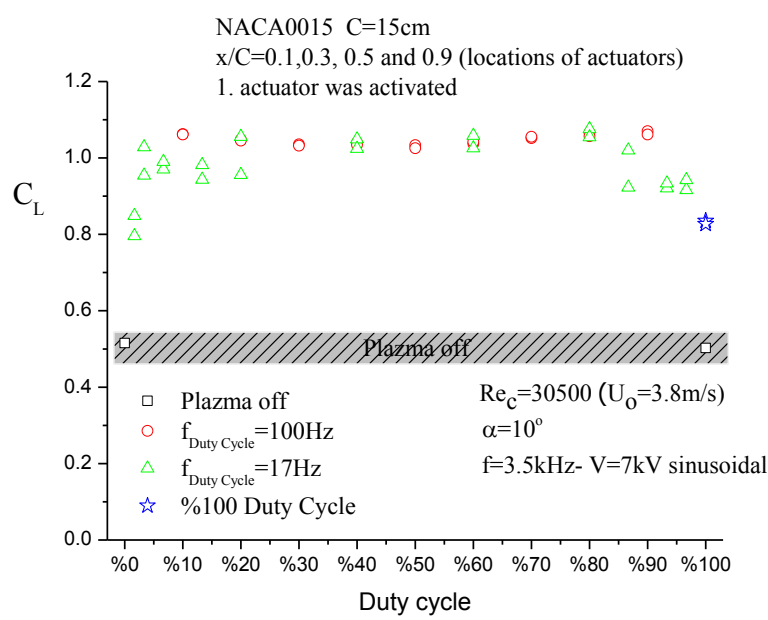

Fig. 11. Effect of percentage of duty cycles on lift coefficient when the plasma was off, excitation frequency $100 \mathrm{~Hz}$ and $17 \mathrm{~Hz}$

In figure 12, the influence of excitation frequency on lift coefficient is given. As it can be seen from the figure, increasing the excitation frequency up to $35 \mathrm{~Hz}$ increased the lift coefficient. There was no chance on the lift coefficient when the excitation frequency was higher than $35 \mathrm{~Hz}$.

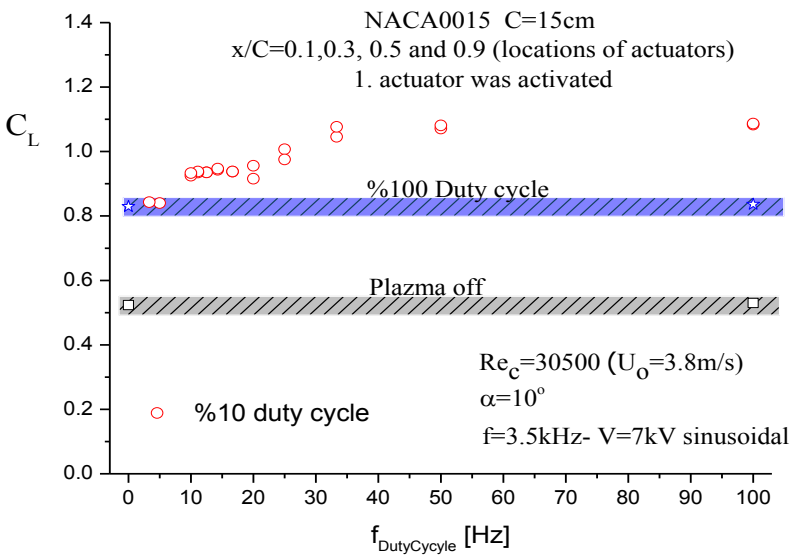

Fig. 12. Effect of excitation frequency to lift coefficient when the plasma was active, passive and \%10 Duty cycle

Flow structure around NACA0015 airfoil geometry was visualized by smoke-wire method at Reynolds number 15000 and 30000. In the case of four actuators excitation, the flow structures around the airfoil at $\alpha=10^{\circ}$ and $\operatorname{Re}_{\mathrm{C}}=$ 30000 were presented in figure 13 for the increments of the voltage. As seen in the figure, the separated shear layer from the leading edge come close to surface by the effect of the plasma. When the driven voltage is increased, the separated shear layer flows closer on the surface causing a higher lift effect.

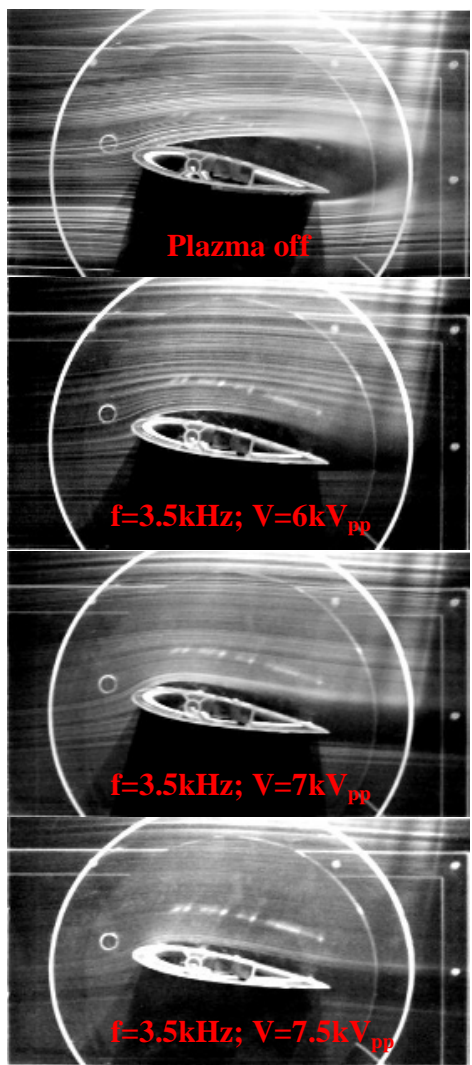

Fig. 13. Effect of voltage on the plasma induced flow structure around NACA0015 airfoil at $\alpha=10^{\circ}$ and $\operatorname{Re}_{C}=30000$ when four actuators were activated. 
The flow structure at different attack angles around the airfoil is seen at flow visualization in figure 14. In this case, the actuator on the location of $\mathrm{x} / \mathrm{C}=0.1$ was activated at $\operatorname{Re}_{\mathrm{C}}=15000$.
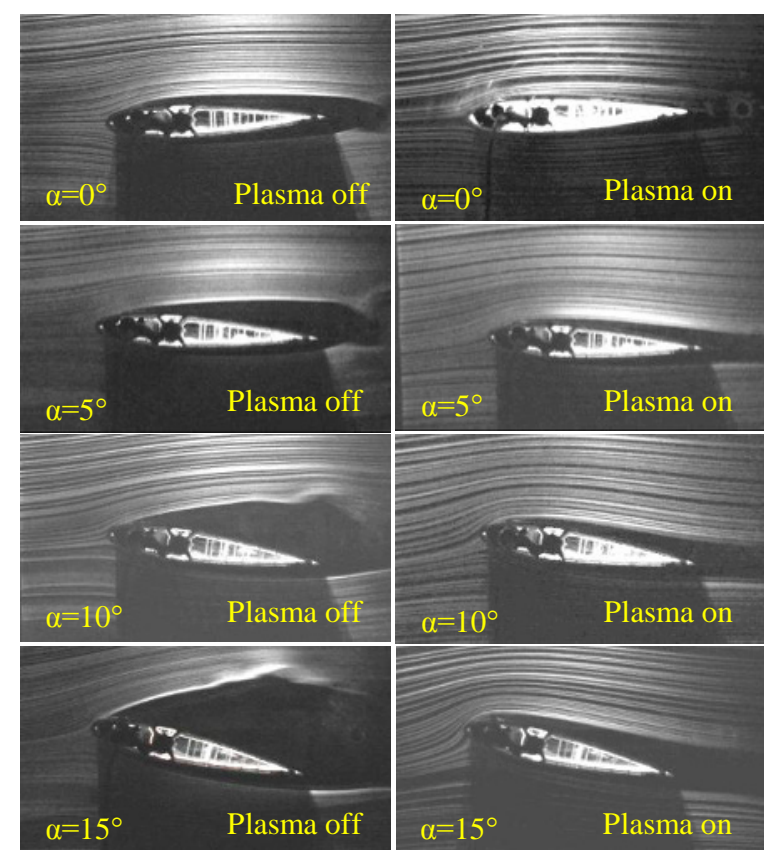

$\alpha=15$

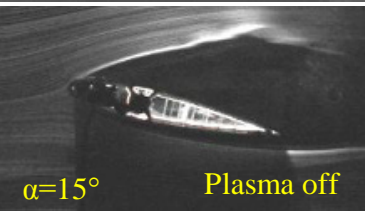

Fig. 14. Effect of voltage to plasma induced flow structure around NACA0015 airfoil at $\mathrm{Re}=15000$

When the angle of attack was $\alpha=0^{\circ}$, growing boundary layer on the airfoil was attached on the surface by the activation of the plasma actuator at $12 \mathrm{kVpp}$ voltage and $4 \mathrm{kHz}$ frequency. By increasing the attack angle, flow separation occurs from the upper surface and the wake region increases gradually at the attack angles of $\alpha=5^{\circ}, \alpha$ $=10^{\circ}, \alpha=15^{\circ}$. When the plasma is on, it is clearly shown that the separated flow attaches and the separation point shifts to downstream. As a result, the streamlines follow the surface of the airfoil. When the attack angle increased to $\alpha=20^{\circ}$, the plasma could not reattach the flow at 12 $\mathrm{kVpp}$ voltage and $4 \mathrm{kHz}$ frequency. When the experiment repeated with the $13 \mathrm{kVpp}$ voltage, the separated flow was controlled as shown in figure 15.

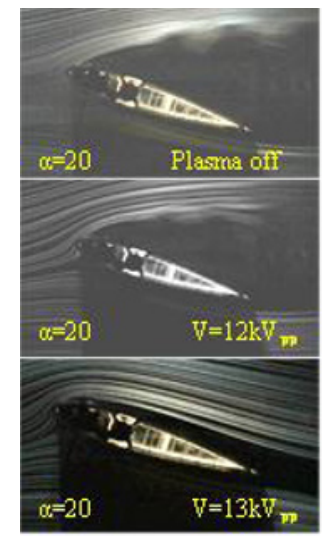

Fig. 15. Flow structures around NACA0015 airfoil at angles of attack $\alpha=0^{\circ}, 5^{\circ}, 10^{\circ}, 15^{\circ}, 20^{\circ}$ at $\mathrm{Re}=15000$ when the actuator was active and passive.

\section{Conclusion}

In this paper, the effect of plasma actuator on a flat plate and manipulation of flow separation on NACA0015 airfoil with plasma actuator at low Reynolds numbers were experimentally investigated. In first case, the effect of voltage, frequency, dielectric thickness, signal manipulation, and measurement location were examined. The result could be summarized as follows:

- The plasma induced velocity profile was increased from $0.8 \mathrm{~m} / \mathrm{s}$ to $2 \mathrm{~m} / \mathrm{s}$ by changing the voltage from 6 to $8 \mathrm{kV}$ peak-peak..

- By increasing the dielectric layer thickness, it was possible to reach higher voltage which results higher induced velocity..

- Excitation of actuator by duty cycle changes the velocity profile. When the duty cycle was $\% 100$ the induced velocity was maximum on the surface like jet flow. By changing the duty cycles, the velocity profile was thicker in $y$ direction and the power consumption was decreased

- The value of induced velocity was also depend on the measurement location. By closing to plasma actuator, the velocity magnitude was increasing.

In second case, the plasma actuator located on NACA0015 airfoil. The lift coefficient of airfoil was examined by changing the attack angle, voltage, frequency, duty cycle rate and excitation frequency. The effect of voltage and attack angle to plasma induced flow structure were visualized and compared with baseline airfoil at $\mathrm{Re}_{\mathrm{C}}=15000$ and 30000 . The results were summarized below;

- The attack angle was shifted from $6^{\circ}$ to $10^{\circ}$ by activating the plasma actuator, and the lift coefficient was increased considerably.

- The lift coefficient was increased by increasing the RF frequency.

- By increasing the voltage, the lift coefficient was increased in cases of single and multiple actuator arrays. At low voltages the multiple actuator array was more effective. However by changing the voltage, the single actuator was inducing the more neutral particles. At highest voltage the effect of multiple and single actuator array to flow were same. As a result, at high voltage the single actuator near the separation point was more efficient.

- It is possible to obtain \%25 lift coefficient increment by activating the actuators by the duty cycle rates between 10-90\% comparing with continuous sinusoidal signal (100\% duty cycle). Moreover, activating the actuators by duty cycle consume less amount of power.

- The flow visualizations showed that by increasing the attack angle, the flow starts to separate from the leading edge of the airfoil. By increasing voltage, the effect of the plasma increases and the separated shear layer flows closer on the surface causing a higher lift effect. 


\section{Acknowledgement}

The authors would like to acknowledge the financial support of this work by the Scientific and Technological Research Council of Turkey (TUBITAK) under the Contract Number of 110M056.

\section{Referances}

1. J. R. Roth, Phys. Plasmas 10, 5 (2003)

2. J. R. Roth, H. Sin, R.C.M Madhan, S.P. Wilkinson, 41st AIAA Aerospace Sciences Meeting, (Reno, 2003)

3. J. R. Roth, R. C. M. Madhan, M. Yadav, J. Rahel, and S. P. Wilkinson, 42nd AIAA Aerospace Sciences Meeting and Exhibit, 845 (Reno, 2004)

4. F.O. Thomas, T.C. Corke, M. Iqbal, A. Kozlov, D. Schatzman, AIAA J. 47, 9 (2009)

5. R. Sosa, G. Artana, J. Electrostat. 64, 604-610 (2006)

6. N. Bernard, E. Moreau, J. Phys. 43, 145201( 2010)

7. J. Little, AIAA J. 48, 12 (2010)

8. N. Benard, J. Jolibois, E. Moreau, J. Electrostat. 67, 133-139 (2009)

9. J. Jolibois, M. Forte, E. Moreau, J. Electrostat. 66, 496-503 (2008)

10. B. Göksel, D. Greenblatt, I. Rechenberg, R. Bannasch, C.O. Paschereit, MAV07 \& EMAV2007, (Toulouse, 2007) 